\title{
Generalized Reciprocal Sanskruti Index: Chemical Applicability and Bounds
}

\author{
Shanmukha M C 1, * (D), Basavarajappa N S ${ }^{2}$, Anil Kumar K N ${ }^{2}$, Shilpa K C ${ }^{3}$ \\ Department of Mathematics, Jain Institute of Technology, Davanagere-577003, Karnataka, India \\ Department of Mathematics, Bapuji Institute of Engineering and Technology, Davanagere-577004, Karnataka, India \\ Department of Computer Science \& Engineering, Bapuji Institute of Engineering and Technology, Davanagere-577004, \\ Karnataka, India \\ * Correspondence: mcshanmukha@gmail.com;
}

Scopus Author ID 57217312072

Received: 30.07.2020; Revised: 15.08.2020; Accepted: 16.08.2020; Published: 19.08.2020

Abstract: In this paper, we put forward the generalization of reciprocal Sanskruti index $\mathcal{R} \mathcal{S}^{\alpha}$ for some $\alpha \in Z^{+}$. For $\alpha=3$ the prediction potentiality of $\mathcal{R} S^{3}$ is discussed with the physicochemical properties of the set of octane isomers. And also we study the mathematical properties of $\mathcal{R} \delta^{3}$.

Keywords: Topological indices; Sanskruti index; Reciprocal Sanskruti index.

(C) 2020 by the authors. This article is an open-access article distributed under the terms and conditions of the Creative Commons Attribution (CC BY) license (https://creativecommons.org/licenses/by/4.0/).

\section{Introduction}

Let $G=(V, E)$ be a simple graph with $|V|=\mathrm{n}$ and $|E|=\mathrm{m}$. Let $\Delta=\operatorname{deg}\left(v_{1}\right) \geq$ $\operatorname{deg}\left(v_{2}\right) \geq \operatorname{deg}\left(v_{3}\right), \ldots, \geq \operatorname{deg}\left(v_{n}\right)=\delta$ be vertex degree sequence and let $\Delta^{\prime}=\sigma\left(v_{1}\right) \geq$ $\sigma\left(v_{2}\right) \geq \sigma\left(v_{3}\right) \geq, \ldots, \sigma\left(v_{n}\right)=\delta^{\prime}$ be neighborhood degree sequence of $\mathrm{G}$ respectively. Where $\sigma(u)=\sum_{v \in N(u)} \operatorname{deg}(v)$ and $N(u)=\{v \backslash u v \in E(G)\}$. S(G) and $\mathrm{L}(\mathrm{G})$ denote the subdivision and line graph of $\mathrm{G}$, respectively.

A Topological Index of a graph is a numerical value that is invariant under the automorphism of graphs. Due to numerous applications in chemistry as a molecular structure descriptors, topological indices gained considerable popularity in the field of mathematical chemistry. These topological indices are used in QSAR/QSPR [1, 4, 12, 16, 17] studies to predict the physicochemical characteristics and biological activities of chemical compounds using a molecular graph. Topological indices can be classified into several distinct categories. One of the widely used group is the so-called degree-based topological indices. A large number of degree-based topological indices have been studied so far [2-10,13-15,18], and references are cited therein.

The most studied topological indices are the Zagreb indices.

$$
\begin{aligned}
& M_{1}(G)=\sum_{v \sim u} d_{G}(v)^{2} \\
& M_{2}(G)=\sum_{v \sim u} d_{G}(v) d_{G}(u)
\end{aligned}
$$

The Sanskruti index [11] is defined as

$$
S(G)=\sum_{v_{i \sim} v_{j}}\left(\frac{v_{i} v_{j}}{v_{i+} v_{j}-2}\right)^{3}
$$

Motivated by the previous research on Sanskruti index, here we propose the following topological indices:

- Generalized Reciprocal Sanskruti Index: 


$$
\mathcal{R S}(G)^{\propto}=\sum_{v_{i \sim} v_{j}}\left(\frac{v_{i+} v_{j}-2}{v_{i} v_{j}}\right)^{\propto}
$$

- $\quad$ For $\propto=1$,

$$
\mathcal{R S}(\mathrm{G})=\sum_{v_{i \sim} v_{j}}\left(\frac{v_{i+} v_{j}-2}{v_{i} v_{j}}\right)
$$

- Randic type reciprocal Sanskruti index:

$$
R T=\sum_{v_{i \sim} v_{j}} \frac{1}{v_{i} v_{j}}
$$

- $\quad$ Reduced Sanskruti index:

$$
R S I=\sum_{v_{i \sim} v_{j}} \frac{v_{i+} v_{j}}{v_{i} v_{j}}
$$

In this paper we are intrested to work on the reciprocal sanskruti index for $\propto=3$. Which is defined as follows:

$$
\mathcal{R S}(G)^{3}=\sum_{v_{i \sim} v_{j}}\left(\frac{v_{i+} v_{j}-2}{v_{i} v_{j}}\right)^{3}
$$

Whenever we introduced a new topological index, it is mandatory to check its applicability in chemistry as a molecular structure descriptor. Therefore, in the following section, we investigate the applicability of $\mathcal{R} \mathcal{S}(G)^{3}$ index.

\section{Materials and Methods}

\subsection{On chemical applicability of the $R S(G)^{\wedge} 3$-Index.}

Here we have examined the chemical applicability of the reciprocal Sanskruti $\mathcal{R} \mathcal{S}(G)^{3}$ - index and compared the values with the Sanskruti $\mathcal{S}(G)$ - index for modeling the physical and chemical properties [boiling points(BP), molar volumes (mv) at $20^{\circ} \mathrm{C}$, molar refractions (mr) at $20^{\circ} \mathrm{C}$, heats of vaporization (hv) at $25^{\circ} \mathrm{C}$, surface tensions (st) $20^{\circ} \mathrm{C}$ and melting points (mp), acentric factor (AcentFac), and DHVAP] of octane isomers. The $\mathcal{R S}(G)^{3}$ was tested using a data set of octane isomers found at (http://www.moleculardescriptors.eu//dataset.htm). The values are compiled in Table 1.

Table 1. Physico-chemical properties of octane isomers.

\begin{tabular}{l|c|c|c|c|c|c|c|c|c|c} 
Alkane & $\mathbf{A F}$ & $\mathbf{D H V A P}$ & $\mathbf{B P}$ & $\mathbf{T C}$ & $\mathbf{P C}$ & $\mathbf{S}$ & $\mathbf{D}$ & $\boldsymbol{R}_{\boldsymbol{m}}^{2}$ & $-\boldsymbol{\Delta}_{\boldsymbol{f}}$ & $-\boldsymbol{\Delta}_{\boldsymbol{v}}$ \\
\hline n-octane & 0.3978 & 9.915 & 125.70 & 296.20 & 24.64 & 111.67 & 0.7025 & 2.0449 & 208.6 & 41.49 \\
\hline 2M & 0.3779 & 9.484 & 117.6 & 288.0 & 24.80 & 109.84 & 0.6980 & 1.8913 & 215.4 & 39.67 \\
\hline 3M & 0.3710 & 9.521 & 118.9 & 292.0 & 25.60 & 111.26 & 0.7058 & 1.7984 & 212.5 & 39.83 \\
\hline 4M & 0.3715 & 9.483 & 117.7 & 290.0 & 25.60 & 109.32 & 0.7046 & 1.7673 & 210.7 & 39.64 \\
\hline 3E & 0.3624 & 9.476 & 118.5 & 292.0 & 25.74 & 109.43 & 0.7136 & 1.7673 & 210.7 & 39.64 \\
\hline 22MM & 0.3394 & 8.915 & 106.8 & 279.0 & 25.60 & 103.42 & 0.6953 & 1.6744 & 224.6 & 37.28 \\
\hline 23MM & 0.3482 & 9.272 & 115.6 & 293.0 & 26.60 & 108.02 & 0.7121 & 1.6464 & 213.8 & 38.78 \\
\hline 24MM & 0.3442 & 9.029 & 109.4 & 282.0 & 25.80 & 106.98 & 0.7004 & 1.6142 & 219.2 & 37.76 \\
\hline 25MM & 0.3568 & 9.051 & 109.1 & 279.0 & 25.00 & 105.72 & 0.6935 & 1.6449 & 222.5 & 37.85 \\
\hline 33MM & 0.3225 & 8.973 & 112.0 & 290.8 & 27.20 & 104.74 & 0.7100 & 1.7377 & 220.0 & 37.53 \\
\hline 34MM & 0.3403 & 9.316 & 117.7 & 298.0 & 27.40 & 106.59 & 0.7200 & 1.5230 & 212.8 & 38.97 \\
\hline 2M3E & 0.3324 & 9.209 & 115.6 & 295.0 & 27.40 & 106.06 & 0.7193 & 1.5525 & 211.0 & 38.52 \\
\hline 3M3E & 0.3068 & 9.081 & 118.3 & 305.0 & 28.90 & 101.48 & 0.7274 & 1.5212 & 214.8 & 37.99 \\
\hline 223MMM & 0.3008 & 8.826 & 109.8 & 294.0 & 28.20 & 101.31 & 0.7161 & 1.4306 & 220.0 & 36.91 \\
\hline 224MMM & 0.3053 & 8.402 & 99.24 & 271.1 & 25.50 & 104.09 & 0.6919 & 1.4010 & 224.0 & 35.14 \\
\hline 233MM & 0.2931 & 8.897 & 114.8 & 303.0 & 29.00 & 102.06 & 0.7262 & 1.4931 & 216.3 & 37.27 \\
\hline 234MMM & 0.3174 & 9.014 & 113.5 & 295.0 & 27.60 & 102.39 & 0.7191 & 1.3698 & 217.3 & 37.75 \\
\hline 2233MMMM & 0.2552 & 8.41 & 106.5 & 270.8 & 24.50 & 93.06 & 0.8242 & 1.4612 & 225.6 & 42.90
\end{tabular}

Surprisingly, we can see that the correlation coefficient value of $\mathcal{R S}(G)^{3}$ for the boiling points of octane, the isomer is $r=0.701$, whereas for $\mathcal{S}(G)$ the correlation coefficient value is $r=0.366$. Similarly for other physical properties of octane isomers such as heats, critical 
temperature, critical pressure, density, entropy and mean radius, the correlation coefficient value for $\mathcal{R S}(G)^{3}$ is $r=0.670,0.473,0.636,0.810$ and 0.687 respectively, where as for $\mathcal{S}(G)$ the correlation coefficient value is $r=0.435,0.010,0.611,0.857$ and 0.669 respectively. Clearly, the reciprocal Sanskruti index $\mathcal{R S}(G)^{3}$ shows a better correlation than $\mathcal{S}(G)$. In Figure 1, the correlation coefficient of $\mathcal{R S}(G)^{3}$ and $\mathrm{S}(\mathrm{G})$ are depicted.
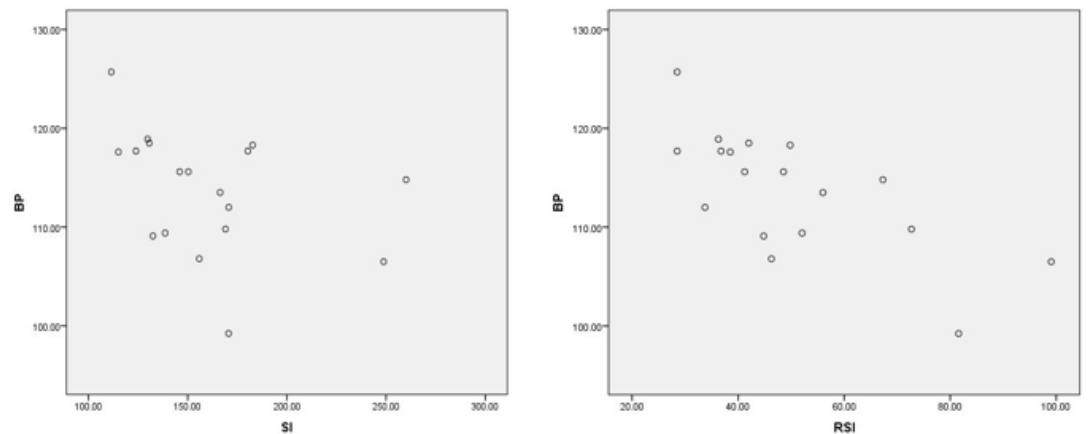

Figure 1. Correlation coefficient.

\section{Results and Discussion}

\subsection{Mathematical properties of $\mathcal{R S}(G)^{3}$.}

Since, $\left(\frac{v_{i+} v_{j}-2}{v_{i} v_{j}}\right)^{3}$ is equal for all edges if a graph $\mathrm{G}$ is regular or biregular. Therefore we have the following axillary results:

Proposition 1. For any r-regular graph $\mathrm{G}, S^{3}(G)=\frac{4 n\left(r^{2}-1\right)^{3}}{r^{11}}$

Proof. Let G be a r-regular graph. Then for every $v \in V(G), v_{j}=r^{2}$. Further, every r-regular graph containing $\frac{n r}{2}$ edges. Therefore we have,

$$
\begin{aligned}
\mathcal{R S}(G)^{3} & =\sum_{v_{i \sim} v_{j}}\left(\frac{v_{i+} v_{j}-2}{v_{i} v_{j}}\right)^{3} \\
& =\frac{n r}{2}\left(\frac{r^{2}+r^{2}-2}{r^{4}}\right)^{3} \\
& =\frac{4 n\left((n-1)^{2}-1\right)^{3}}{r^{11}}
\end{aligned}
$$

\section{Proposition 2.}

(a) For a complete graph $K_{n}, S^{3}\left(K_{n}\right)=\frac{4 n\left((n-1)^{2}-1\right)^{3}}{(n-1)^{11}}$

(b) For cycle graph $C_{n}, S^{3}\left(C_{n}\right)=\frac{27 n}{512}$

(c) For path graph $P_{n}, S^{3}\left(P_{n}\right)=\frac{27 n+47}{512}$

(d) For the wheel graph $W_{n}, S^{3}\left(W_{n}\right)=(n-1)\left[\left(\frac{4 n-6}{3(n-1)^{2}}\right)^{3}+\left(\frac{n+8}{n+5}\right)^{3}\right]$

(e) For complete biparatite graph $K_{r, s} ; 2 \leq r<s, S^{3}\left(K_{r, s}\right)=\frac{8(r s-1)^{3}}{r s^{5}}$

Proof. (a) and (b) follows by Proposition 1, putting $\mathrm{r}=\mathrm{n}-1$ and $\mathrm{r}=2$ respectively.

(c) Let $\mathrm{V}\left(\mathrm{P}_{\mathrm{n}}\right)=\left\{\mathrm{v}_{1}, \mathrm{v}_{2}, \mathrm{v}_{3}, \ldots . ., \mathrm{V}_{\mathrm{n}}\right\}$ where $\mathrm{v}_{1}$ and $\mathrm{v}_{\mathrm{n}}$ are pendent vertices of $\mathrm{P}_{\mathrm{n}}$. Then for every $v \in$ $V\left\{v_{1}, v_{n}\right\}, v_{j}=4$ and $\sigma\left(v_{1}\right)=5=\sigma\left(v_{n}\right)$. Since $E\left(P_{n}\right)=n-1$, therefore, we have 


$$
\begin{gathered}
\mathcal{R S}\left(P_{n}\right)^{3}=\sum_{v_{i \sim} v_{j}}\left(\frac{v_{i+} v_{j}-2}{v_{i} v_{j}}\right)^{3} \\
=(\mathrm{n}-3)\left(\frac{4+4-2}{4^{2}}\right)^{3}+2\left(\frac{2+3-2}{2 \times 3}\right)^{3} \\
=\frac{27 n+47}{512}
\end{gathered}
$$

(d) Let $\mathrm{V}\left(\mathrm{W}_{\mathrm{n}}\right)=\left\{\mathrm{v}_{1}, \mathrm{v}_{2}, \mathrm{v}_{3}, \ldots \ldots, \mathrm{V}_{\mathrm{n}}\right\}$ where $\mathrm{v}_{1}$ is a central vertex of $\mathrm{W}_{\mathrm{n}}$. Then for every $v \in$ $V \backslash\left\{v_{1}\right\}, v_{j}=n+5$ and $\sigma\left(v_{1}\right)=3(n-1)$. Since $E\left(W_{n}\right)=2(n-1)$, therefore, we have

$$
\begin{gathered}
\mathcal{R} \mathcal{S}\left(W_{n}\right)^{3}=\sum_{v_{i \sim} v_{j}}\left(\frac{v_{i+} v_{j}-2}{v_{i} v_{j}}\right)^{3} \\
=(n-1)\left(\frac{3(n-1)+(n-1)-2}{3(n-1)^{2}}\right)^{3}+(n-1)\left(\frac{(n+5)+(n+5)-2}{(n+5)^{2}}\right)^{3} \\
=(n-1)\left[\left(\frac{4 n-6}{3(n-1)^{2}}\right)^{3}\left(\frac{n+8}{n+5}\right)^{3}\right]
\end{gathered}
$$

(e) Let $V\left(K_{r, s}\right)=v_{1} \cup v_{2}$ where $v_{1}=\left\{v_{1}, v_{2}, v_{3}, \ldots, v_{r}\right\}$ and $v_{2}=\left\{v_{r+1}, v_{r+2}, v_{r+3}, \ldots, v_{s}\right\}$. Clearly, every $v \in v_{1}, v_{j}=r s$ and $u \in v_{2}, v_{i}=s r$. Since $E\left(K_{r, s}\right)=r s$

therefore, we have

$$
\begin{aligned}
\mathcal{R S}\left(K_{r, s}\right)^{3} & =\sum_{v_{i \sim} v_{j}}\left(\frac{v_{i+} v_{j}-2}{v_{i} v_{j}}\right)^{3} \\
= & r s\left(\frac{r s+s r-2)}{r s^{2}}\right)^{3} \\
& =\frac{8(r s-1)^{3}}{r s^{5}}
\end{aligned}
$$

We need the following well-known results to obtain bounds for $R S(G)^{3}$.

Lemma 1 ([19]). Let $a_{i}, i=1,2,3, \ldots, m$ be a positive real number sequence. Then for any real $r, r \geq 1$ or $r \leq 0$, the following inequality holds

$\sum_{i=1}^{m} a_{i}^{r} \geq m^{1-r}\left(\sum_{i=1}^{m} a_{i}\right)$.

Equality holds if and only if $a_{1}=a_{2}=\cdots=a_{m}$.

Lemma 2 ([20]). Let $a_{i}$ and $b_{i}, i=1,2,3, \ldots, m$ be a positive real number sequences. Then for any real $r \geq 0$ the following inequality holds

$\sum_{i=1}^{m} \frac{a_{i}^{r+1}}{b_{i}^{r}} \geq \frac{\left(\sum_{i=1}^{m} a_{i}\right)^{r+1}}{\left(\sum_{i=1}^{m} b_{i}\right)^{r}}$

Equality holds if and only if $\frac{a_{1}}{b_{1}}=\frac{a_{2}}{b_{2}}=\cdots=\frac{a_{m}}{b_{m}}$.

\subsection{Bounds for $R S(G)^{3}$.}

Next, we have the following lower bound for $R S(G)^{3}$ in terms of maximum degree and the size of a graph $\mathrm{G}$.

Theorem 3. For any graph $\mathrm{G}$ without isolated vertices,

$$
R S(G)^{3} \geq 8 m\left(\frac{1}{\Delta^{2}}-\frac{1}{\Delta^{4}}\right)^{3}
$$

Proof. Let $\mathrm{G}$ be graph without isolated vertices of size $\mathrm{m}$ and maximum degree $\Delta$. Since for any $v \in V, v_{j} \leq \Delta^{2}$. Therefore, we have,

$$
R S(G)^{3}=\sum_{v_{i \sim} v_{j}}\left(\frac{v_{i+} v_{j}-2}{v_{i} v_{j}}\right)^{3} \geq m\left(\frac{\Delta^{2}+\Delta^{2}-2}{\Delta^{4}}\right)^{3}
$$




$$
\begin{aligned}
& =8 m\left(\frac{\Delta^{2}-1}{\Delta^{4}}\right)^{3} \\
& =8 m\left(\frac{1}{\Delta^{2}}-\frac{1}{\Delta^{4}}\right)^{3}
\end{aligned}
$$

In the following results, we obtain lower bound for $R S(G)^{3}$ in terms of maximum degree $\Delta$.

Theorem 4. For any n-vertex graph $\mathrm{G}$ and maximum degree $\Delta$,we have

$$
R S(G)^{3} \geq \frac{8\left(\Delta^{4}-1\right)^{3}}{\Delta^{12}\left(\Delta^{2}+1\right)^{3}}
$$

Proof. Let $\mathrm{G}$ be a n-vertex graph with a maximum degree $\Delta$, we have

$$
\begin{aligned}
& \mathcal{R} \mathcal{S}(G)^{3}=\sum_{v_{i \sim} v_{j}}\left(\frac{v_{i+} v_{j}-2}{v_{i} v_{j}}\right)^{3} \\
& =\sum_{v_{i \sim} v_{j}}\left(\frac{v_{i} v_{j}-1}{v_{i} v_{j}}\right)^{3}\left(\frac{v_{i+} v_{j}-2}{v_{i} v_{j}-1}\right)^{3}
\end{aligned}
$$

Since $\left(v_{i}-v_{j}\right)^{2} \geq 0$, therefore it is easy to check that

$$
\frac{v_{i+} v_{j}-2}{v_{i} v_{j}-1}=\frac{1}{v_{i}+1}+\frac{1}{v_{j}+1} \text {. }
$$

Since $v_{i} \leq \Delta^{2}$ therefore (15) becomes

$$
\frac{v_{i+} v_{j}-2}{v_{i} v_{j}-1}=\frac{1}{\Delta^{2}+1}+\frac{1}{\Delta^{2}+1}
$$

Employing (16) in (14), we get

$$
\begin{aligned}
R S(G)^{3} & \geq\left(\frac{\Delta^{2} \Delta^{2}-1}{\Delta^{4}}\right)^{3}\left(\frac{2}{\Delta^{2}+1}\right)^{3} \\
& =\frac{8\left(\Delta^{4}-1\right)^{3}}{\Delta^{12}\left(\Delta^{2}+1\right)^{3}}
\end{aligned}
$$

Theorem 5. Let $\mathrm{G}$ be a graph without isolated vertex. Then

$$
R S(G)^{3} \geq \frac{(R S I-2 R T)^{3}}{m^{2}}
$$

Proof. Let $\mathrm{G}$ be a graph without isolated vertex. Then set $r=3, a_{i}=\frac{v_{i+} v_{j}-2}{v_{i} v_{j}}$ where the summation is performed over all edges of $\mathrm{G}$ then (9) is transformed into

$$
\begin{gathered}
\sum_{v_{i \sim} v_{j}}\left(\frac{v_{i+} v_{j}-2}{v_{i} v_{j}}\right)^{3} \geq m^{1-3} \sum_{v_{i \sim} v_{j}}\left(\frac{v_{i+} v_{j}-2}{v_{i} v_{j}}\right)^{3} \\
\geq\left(\sum_{v_{i \sim} v_{j}} \frac{v_{i+} v_{j}}{v_{i} v_{j}}-\sum_{v_{i \sim} v_{j}} \frac{2}{v_{i} v_{j}}\right)^{3} \\
=\frac{(R S I-2 R T)^{3}}{m^{2}}
\end{gathered}
$$

Theorem 6. Let $\mathrm{G}$ be a graph without isolated vertex with size at least 2 .

Then

$$
R S(G)^{3} \leq\left(2 M_{2}-2 m\right)^{3}(R T)^{3}
$$

Proof. Observe that the following identity holds

$$
\begin{gathered}
2 M_{2}-2 m=\sum_{v_{i \sim} v_{j}}\left(v_{i+} v_{j}\right)-2 m \\
=\sum_{v_{i \sim} v_{j}} v_{i+} v_{j}-2
\end{gathered}
$$




$$
\left(2 M_{2}-2 m\right)^{3}=\sum_{v_{i \sim} v_{j}} \frac{\left(\frac{v_{i+} v_{j}-2}{v_{i} v_{j}}\right)^{3}}{\left(\frac{1}{v_{i} v_{j}}\right)^{3}}
$$

Set $r=2, a_{i}=\frac{v_{i+} v_{j}-2}{v_{i} v_{j}}$ and $b_{i}=\frac{1}{v_{i} v_{j}}$ in $(10)$, we get

\section{Conclusions}

$$
\begin{aligned}
& \sum_{v_{i} v_{j}} \frac{\left(\frac{v_{i+} v_{j}-2}{v_{i} v_{j}}\right)^{3}}{\left(\frac{1}{v_{i} v_{j}}\right)^{3}} \times \frac{1}{v_{i} v_{j}} \geq \frac{\left(\sum_{v_{i \sim} v_{j}}\left(\frac{1}{v_{i} v_{j}}\right)\right)^{3}}{\left(\sum_{v_{i \sim} v_{j}} \frac{1}{v_{i} v_{j}}\right)^{2}} \\
&\left(2 M_{2}-2 m\right)^{3} R T \geq \frac{R S(G)^{3}}{(R T)^{\epsilon}} \\
& R S(G)^{3} \leq\left(2 M_{2}-2 m\right)^{3}(R T)^{3}
\end{aligned}
$$

Inspired by the work on the Sanskruti index, the reciprocal Sanskruti index is proposed. The value of the reciprocal Sanskruti index is calculated for octane isomers. The chemical applicability is compared to model the physical and chemical properties of octane isomers. The reciprocal Sanskruti index gives better results than the Sanskruti index.

\section{Funding}

This research received no external funding.

\section{Acknowledgments}

This research has no acknowledgment.

\section{Conflicts of Interest}

\section{The authors declare no conflict of interest.}

\section{References}

1. Anil Kumar, K.N.; Basavarajappa, N.S.; Shanmukha, M.C. QSPR analysis of Alkanes with certain degree based topological indices. Malaya Journal of Mathematik 2020, 8, 314-330, https://doi.org/10.26637/MJM0802/0003.

2. Estrada, E. Atom-bond connectivity and the energetic of branched alkanes. Chemical Physics Letters $\mathbf{2 0 0 8 ,}$ 463, 422-425, https://doi.org/10.1016/j.cplett.2008.08.074.

3. Fath-Tabar, G. Old and New Zagreb Indices of Graphs. MATCH - Communications in Mathematical and in Computer Chemistry 2011, 65, 79-84.

4. Randic, M. Quantitative Structure-Property Relationship: boiling points and planar benzenoids. New. j. Chem. 1996, 20, 1001-1009.

5. Furtula, B.; Graovac, A.; Vukičevic', D.; Augmented Zagreb index. Journal of Mathematical Chemistry 2010, 48, 370-380.

6. Aslam, A.; Bashir, Y.; Ahmad, S.; Gao, W. On Topological Indices of Certain Dendrimer Structures. ADS 2017, 72, 559-566, https://doi.org/10.1515/zna-2017-0081.

7. Gutman, I. Selected properties of the Schultz molecular topological index. Journal of Chemical Information and Computer Sciences 1994, 34, 1087-1089, https://doi.org/10.1021/ci00021a009.

8. Gutman, I.; Furtula, B.; Vukićević, Ž.; Popivoda, G. On Zagreb Indices and Coindices. Match 2015, 74, 516.

9. Harary, F. Graph Theory. Addison-Wesely, Reading Mass 1969.

10. Hosamani, S.M.; Basavanagoud, B.; New upper bounds for the first Zagreb index. MATCH Commun. Math. Comput. Chem. 2015, 74, 97-101. 
11. Hosamani, S.M. Computing Sanskruti index of certain nanostructures. Journal of Applied Mathematics and Computing 2017, 54, 425-433, https://doi.org/10.1007/s12190-016-1016-9.

12. Hosamani, S.; Perigidad, D.; Jamagoud, S.; Maled, Y.; Gavade, S. QSPR Analysis of Certain Degree Based Topological Indices. Journal of Statistics Applications \& Probability 2017, 6, 1-11, https://doi.org/10.18576/jsap/060211.

13. Liu, B.; Gutman, I. Upper bounds for Zagreb indices of connected graphs. MATCH - Communications in Mathematical and in Computer Chemistry 2006, 55, 439-446.

14. Hayat, S.; Imran, M.; Liu, J.-B. Correlation between the Estrada index and $\pi$-electronic energies for benzenoid hydrocarbons with applications to boron nanotubes. International Journal of Quantum Chemistry 2019, 119, https://doi.org/10.1002/qua.26016.

15. Chen, J.; Liu, J.; Guo, X. Some upper bounds for the atom-bond connectivity index of graphs. Applied Mathematics Letters 2012, 25, 1077-1081, https://doi.org/10.1016/j.aml.2012.03.021.

16. Shanmukha, M.C.; Basavarajappa, N.S.; Anilkumar, K.N. Predicting physicochemical properties of octane isomers using QSPR approach. Malaya Journal of Matematik 2020, 8, 104-116.

17. Shanmukha, M.C.; Basavarajappa, N.S.; Shilpa, K.C.; Usha, A. Degree-based topological indices on anticancer drugs with QSPR analysis. Heliyon 2020, 6, 1-9, https://doi.org/10.1016/j.heliyon.2020.e04235.

18. Usha, A.; Ranjini, P.S.; Lokesha, V.; Harish. Bounds of Harmonic index. Contemporary Studies in Discrete Mathematics 2018, 2, 1-4.

19. Nadeem, M.F.; Zafar, S.; Zahid, Z. On topological properties of the line graphs of subdivision graphs of certain nanostructures. Applied Mathematics and Computation 2016, 273, 125-130, https://doi.org/10.1016/j.amc.2015.10.010.

20. Ranjini, P.S.; Veera, L.; Rajan, M.A..; Raju, M.P. On the Shultz index of the subdivision graphs. Advanced Studies in Contemporary Mathematics (Kyungshang) 2011, 3, 279-290. 